\title{
A Case of a Giant Cell Tumor of the Tendon Sheath of the Middle Phalanx of the Fourth Toe
}

\author{
Kyochika Okabe, Toshihiko Hoashi, Mariko Asahara, Eiko Esaki, \\ Michiko Ito, Rui Otsuka, Yoko Funasaka and Hidehisa Saeki \\ Department of Dermatology, Nippon Medical School, Tokyo, Japan
}

\begin{abstract}
Giant cell tumors of the tendon sheath (GCT-TS) are one of the most common tumors of the hand, along with ganglions. However, occurrence in the toes is far less common. Here we present a rare case of GCT-TS at the middle phalanx level of the flexor digitorum longus tendon sheath. Magnetic resonance imaging showed low intensity both on $\mathrm{T} 1$ and $\mathrm{T} 2$ weighted images. To the best of our knowledge, our case is the first report of a GCT-TS arising at the middle phalanx level of the flexor digitorum longus tendon sheath of the fourth toe. Although GCT-TS are rare in the toe, they must be included in the differential diagnoses of soft tissue tumors arising in the toes. (J Nippon Med Sch 2017; 84: 308-310)
\end{abstract}

Key words: giant cell tumor of tendon sheath, toe, middle phalanx, surgery, flexor digitorum longus tendon sheath

\section{Introduction}

Giant cell tumors of the tendon sheath (GCT-TS) are one of the most common tumors of the hand, along with ganglions ${ }^{1}$. However, occurrence in the foot is far less common ${ }^{2}$. Here we present a rare case of a GCT-TS at the middle phalanx level of the flexor digitorum longus tendon sheath.

\section{Case Report}

A 31-year-old woman presented with a swelling of the root of her fourth toe, which had been gradually growing for five years without pain. The patient had no apparent history of trauma. On examination, an elastic soft nontender subcutaneous mass sized $25 \mathrm{~mm}$ surrounded almost the entire root of her fourth toe (Fig. 1A and B). There was no mobility of the subcutaneous tissue. Magnetic resonance imaging (MRI) showed low intensity both in T1 and T2 weighted images (data not shown). There was no evidence of cortical erosion. The patient underwent excision of the tumor under general anesthesia using a tourniquet to keep the area avascular in order to dissect the tumor meticulously. Intraoperatively, a soft tissue mass was linked to the flexor digitorum longus tendon and its sheath (Fig. 2A). Histologically, the tumor was encapsulated by a fibrous membrane (Fig. 2B). The tumor was composed of histiocyte-like cells, foam cells, and a mixture of multinucleated giant cells and hemosiderin-laden cells with collagenous stromal fragments. These findings were consistent with a GCT-TS. After six months, there was no sign of local recurrence or any complaint about numbness or flexion disturbance.

\section{Discussion}

A GCT-TS is a benign tumor arising from the synovial membrane. Histological characteristics of GCT-TS are the accumulation of histiocytes and the presence of multinucleated giant cells, foamy cells, and hemosiderin-laden macrophages ${ }^{1}$. The tumor is usually found in women in the third to fifth decade ${ }^{1}$ of life. Ninety percent of GCTTS cases are in the hands or in the fingers. Consequently, those in the foot or toes are far less common'. There are few reports describing GCT-TS in the toes. Ushijima et al. reported that 183 lesions of GCT-TS occurred in the digits and that only 25 of them occurred in the toes ${ }^{3}$. Zhang et al. studied 12 cases of GCT-TS in the foot ${ }^{4}$. Of these 12 cases, five were in the second toe and two were in the hallux. They concluded that GCT-TS commonly arose in the forefoot and hindfoot, especially in the second toe.

Correspondence to Toshihiko Hoashi, MD, PhD, Department of Dermatology, Nippon Medical School, 1-1-5 Sendagi, Bunkyoku, Tokyo 113-8603, Japan

E-mail: thoashi-tky@umin.ac.jp

Journal Website (http://www2.nms.ac.jp/jnms/) 

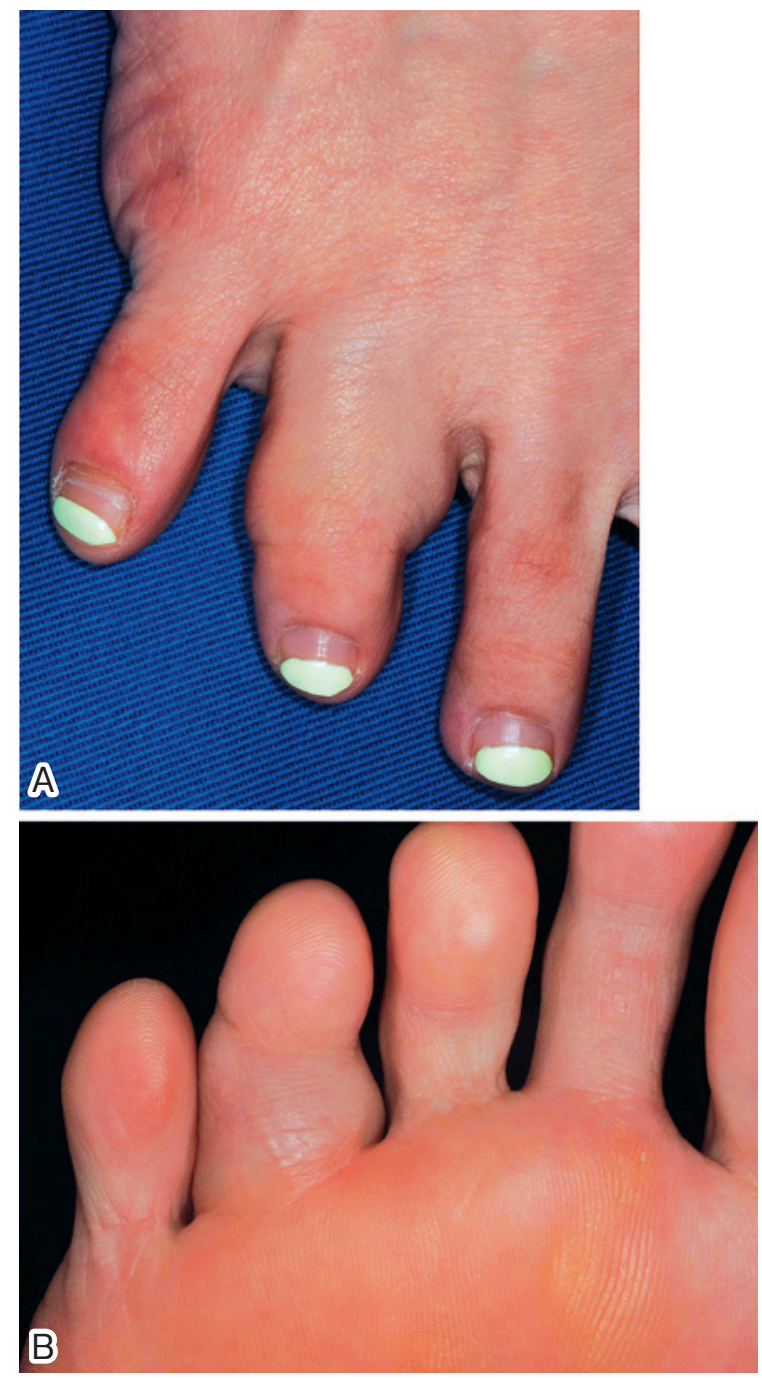

Fig. 1 Clinical features of the giant cell tumor of the tendon sheath of the fourth toe.

Giant cell tumor of the tendon sheath of the fourth toe, preoperative dorsal (a) and volar (b) aspects of the fourth toe.

Gibbons et al. also reported 13 cases of GCT-TS in the foot $^{5}$. Of these 13 cases, four were in the hallux, three in the second toe, one in the third toe, and three in the fifth toe. No case was reported in the fourth toe or at the middle phalanx level. To the best of our knowledge, our case is the first report of a GCT-TS arising at the middle phalanx level of the flexor digitorum longus tendon sheath in the fourth toe. The MRI features of GCT-TS are highly characteristic because T1 and T2 weighted images show low intensity ${ }^{2,5}$. MRI helps in differentiating GCT-TS from other soft tissue tumors, including lipoma and ganglions, which are sometimes observed at the middle phalanx level. Although GCT-TS are rare in the toe, GCT-TS must be included in the differential diagnoses of soft tissue tumors arising in the toes.
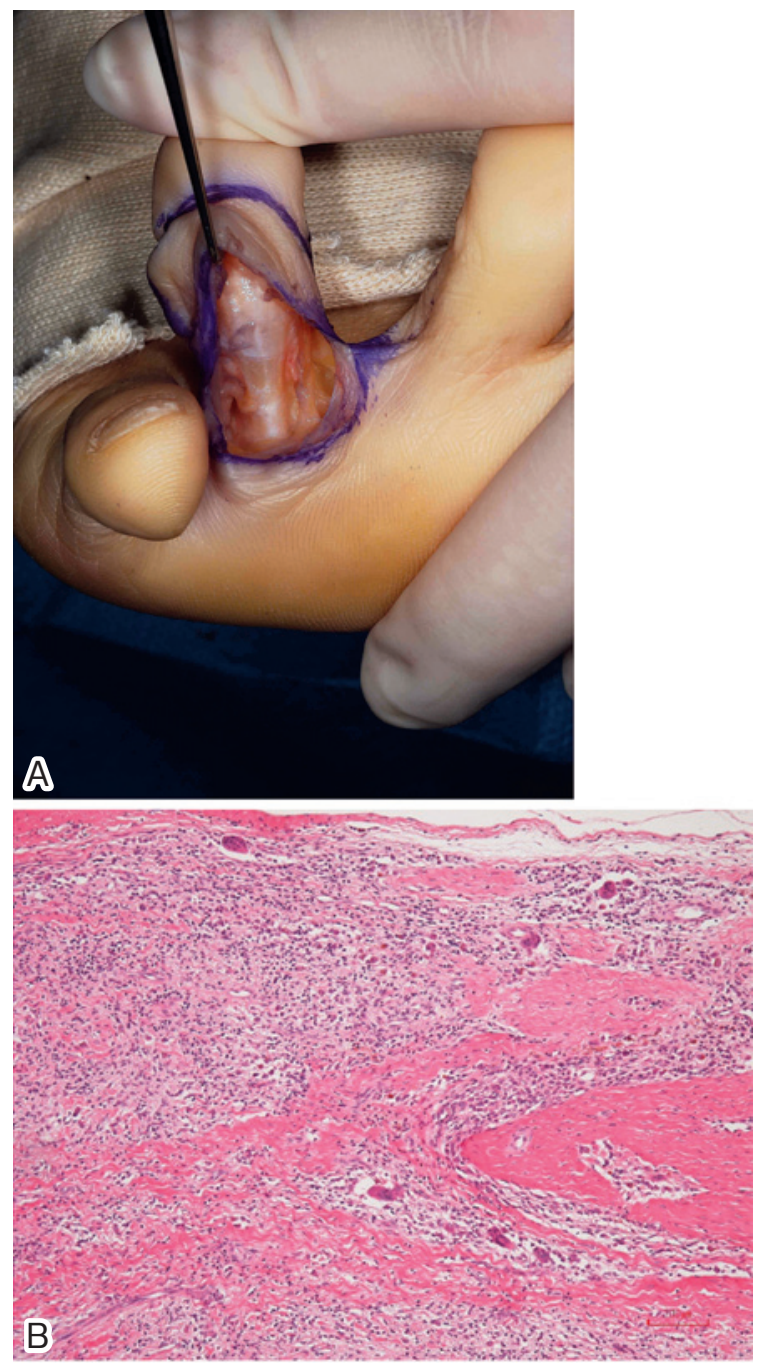

Fig. 2 Intraoperative and histological features of the giant cell tumor of the tendon sheath of the fourth toe.

(a) The flexor digitorum longus tendon was exposed at the operation.

(b) A subcutaneous mass was capsulated by fibrous membrane. The tumor was composed of histiocyte-like cells, foam cells and a mixture of multinucleated giant cells and hemosiderin-laden cells $(\mathrm{HE} \times 100)$.

Acknowledgement: None.

\section{Conflict of Interest: None.}

\section{References}

1. Briet JP, Becker SJ, Oosterhoff T, Ring D: Giant cell tumor of tendon sheath. Arch Bone Jt Surg 2015; 3: 19-21.

2. Patel AS, Thaker P, Patel R, Ladumore M, Prabhakar MM: A rare case report of giant cell tumor of a tendon sheath. Int J Med Sci Public Health 2016; 5: 829-831.

3. Ushijima M, Hashimoto H, Tsuneyoshi M, Enjoji M: Giant cell tumor of the tendon sheath (nodular tenosynovitis). A study of 207 cases to compare the large joint group with the common digit group. Cancer 1986; 57: 875-884. 
4. Zhang Y, Huang J, Ma X, Wang X, Zhang C, Chen L: Giant cell tumor of the tendon sheath in the foot and ankle: case series and review of the literature. J Foot Ankle Surg 2013; 52: 24-27.

5. Gibbons CL, Khwaja HA, Cole AS, Cooke PH, Athanasou NA: Giant-cell tumour of the tendon sheath in the foot and ankle. J Bone Joint Surg Br 2002; 84: 1000-1003.

(Received, August 18, 2017)

(Accepted, August 23, 2017) 